\title{
Functional outcome of ligament reconstruction with tendon interposition after failed first carpometacarpal joint prosthesis
}

\author{
Lyne Anthonissen, Elke Van Eynde, Maarten Van Nuffel, Luc De Smet
}

From the Department of Orthopedic Surgery, Hand Unit, KUL University Hospitals, Leuven, Belgium

The purpose is to determine if ligament reconstruction with tendon interposition (LRTI) is a recommendable salvage option for failed total joint prosthesis of the first carpometacarpal joint.

Twenty-two patients in our database met the inclusion criteria for this retrospective study, with at least 6 months follow-up. Fourteen participated and were invited for a clinical examination and asked to fill out two questionnaires. They were evaluated for pain (VAS), impairment (NHS), disability (Quick DASH), opposition (Kapandji test) and grip strength (hydraulic dynamometer). Results of the questionnaires were compared to a cohort study of primary LRTI's. Kapandji test and grip strength were compared to the contralateral side.

Compared to primary LRTI's, revision surgery showed mild deterioration of impairment and disability. The average VAS score was 2.9 out of 10 . Twelve patients mentioned a sense of strength loss, which could be quantified with the dynamometer: a mean of $15.1 \mathrm{~kg}$ (operated thumb) versus $20.5 \mathrm{~kg}$ (contralateral). There was a relatively small decline of opposition with Kapandji 8.6 versus 9.9. The overall satisfaction was good for 8 patients, fair for 3 and poor for the remaining 3 (mainly based on strength loss). One patient needed a second revision.

Failed first carpometacarpal joint replacement can be salvaged by ligament reconstruction with tendon interposition, providing an acceptable functional outcome in $79 \%$ of cases studied. However, compared to the functional outcome of primary LRTI's, mild aggravation of impairment and disability should be taken into account.

Ethical Approval : Approval from the medical ethics committee from the UZ KU Leuven was granted on 19 October 2015.
Keywords : thumb ; LRTI ; first carpometacarpal joint prosthesis ; revision; strength loss.

\section{INTRODUCTION}

There are several surgical treatments for trapeziometacarpal joint osteoarthritis, one of them being a total joint replacement. Although this procedure is one of the most successful solutions for osteoarthritis of the thumb (1), shortand long-term complications can occur such as peritrapezial joint arthritis, implant subluxation or dislocation, loosening, premature wear, implant fragmentation or foreign body reaction (2). In some cases a re-operation can be performed, removing the prosthesis and preforming a trapeziectomy and ligament reconstruction with tendon interposition (LRTI). In this procedure the flexor carpi radialis tendon is used to stabilize the base of the thumb

\footnotetext{
- Lyne Anthonissen ${ }^{1}, \mathrm{MD}$

Elke Van Eynde², MD

Maarten Van Nuffel, MD

- Luc De Smet ${ }^{3}, \mathrm{MD}, \mathrm{PhD}$

${ }^{1}$ University Hospital Brussels

${ }^{2}$ Sint-Vincentius Hospital Antwerp

${ }^{3} U Z$ Leuven

Correspondence : Lyne Anthonissen, Helenalei 21, 2018 Antwerpen. Phone : +32 494/90.64.41.

Email : Lyne_anthonissen@hotmail.com

- 2021, Acta Orthopædica Belgica.
} 
Table I. Results comparing LRTI after failed total joint prosthesis to primary LRTI (cohort study).

*Statistically significant with $\mathrm{P}<0.05$

\begin{tabular}{|l|c|c|c|c|c|}
\hline & LRTI after prosthesis & \multicolumn{2}{c|}{ Primary LRTI } & P value* \\
\hline & Mean & SD & Mean & SD & T-test unpaired \\
\hline Numbers of patients & 14 & & 179 & & \\
\hline Number of thumbs & 14 & & 233 & & \\
\hline Age & 63.7 & 10.1 & $60.0 \mathrm{y}$ & 7.9 & 0.1 \\
\hline Follow up & $5.9 \mathrm{y}(1-15)$ & & $1.0 \mathrm{y}$ & & \\
\hline Quick DASH & 36.4 & 21.3 & 23.0 & 11.1 & $\underline{0.0001}$ \\
\hline NHS & 63.7 & 23.6 & 75.5 & 21.2 & $\underline{0.044}$ \\
\hline
\end{tabular}

metacarpal and fill the trapeziectomy void. Longterm studies have shown LRTI and total joint prosthesis to be equally successful in the treatment of carpalmetacarpal (CMC) osteoarthritis (3), despite the known complications of LRTI such as subluxation of the metacarpal base and loss of height of the arthroplasty space $(4,5)$.

The main goal of this study is to determine whether a failed prosthesis can be successfully salvaged by an interposition arthroplasty with ligament reconstruction. This will help to establisch that a total joint prosthesis is still a valuable first choice for surgical treatment for CMC arthritis, taking into consideration its faster rehabilitation and the possibility to be revised with LRTI (6).

\section{PATIENTS AND METHODS}

For this retrospective, monocentric, cohort study we retrieved 22 patients from our database between January 1998 and September 2015 that met the inclusion criteria. They all had a failed CMC I prosthesis revised to a LRTI at least 6 months ago. Two patients were excluded because of a poor general condition due to non-related medical conditions (acute myocardial infarction and chemotherapy). Six others were lost to follow-up.

The remaining 14 patients were invited for clinical examination and were asked to fill out the questionnaires. The group consisted of 13 women and 1 male and were all evaluated by the same examiner, independent of the operating surgeon. There were no bilateral revision cases. However 9 out of 14 patients had a LRTI, prosthesis or conservatively treated arthritis of the contralateral CMC I joint.

For the control cohort group of primary LRTI's, we retrieved from 179 patients from the database in the same center, operated between Jan 2000 and Dec 2010 (Table I). The operations where performed by two of the three hand surgeons who did the revision surgeries.

(Appendix A) The main reason for revision was loosening of the cup in the trapezium. This occurred in 7 out the 14 patients, followed by dislocation in 2 patients, peritrapezial joint arthritis in 2, constant pain in 2 and a periprosthetic fracture in one patient. Three different kind of prostheses were removed: 9 Roseland $\AA$ (DePuy, Leeds, England), 3 De La Caffinière ${ }^{\circledR}$ (Stryker, Howmedica) and 2 ARPE ${ }^{\circledR}$ (Zimmer-Biomet Warsaw, IN, USA) prostheses. The average time between the initial operation and the revision was 1.6 years. There were no exclusion criteria concerning the initial placement of the total joint prosthesis. The revisions were all preformed in the same hospital by three different hand surgeons using the same surgical technique.

One of the patients included in this study needed a second revision with a Brunelli procedure (placing the extensor carpi radialis tendon between CMC II and I) due to continuous pain.

After removal of the prosthesis an interposition arthroplasty with ligament reconstruction was performed. The most common technique in our department was a modification of the technique described by Burton and Pellegrini (Fig. 1) (7). In this procedure the flexor carpi radialis (FCR) tendon is divided proximally at two thirds and 
the distal insertion remains intact. The tendon is then fixed through a bone tunnel at the base of the first metacarpal and coiled to fill the void of the trapeziectomy (8). However in 3 cases the FCR was insufficient, resulting in the need for another tendon. Two of them had the Weilby procedure (9) where the adductor pollicis longus was used to reinforce the FCR. In the third patient the extensor carpi radialis longus was used for substitution. Decisions regarding the choice of tendon were left to the discretion of the operating surgeon.

Postoperatively, all patients were given a bulky dressing and were recommended to mobilize the thumb immediately. They were not allowed to exert power for the first 6 to 8 weeks.

During clinical examination grip strength and instability of CMC I where evaluated and the Kapandji score was used to grade the opposition of the thumb from 0 to 10 (10). Grip strength results with the hydraulic Jamar dynamometer (Asimov Engineering Company, Santa Fe Spring, CA) and the Kapandji score were compared to the results of the contralateral thumb. The questionnaires included the Quick DASH score (Disability of Arm, Shoulder and Hand) from 0 to 100 , the Nelson Hospital Score (11) (NHS) for impairment from 100 to 0 and visual analogue scores (VAS) for pain on a scale of 10 .

Secondary endpoints contained the overall satisfaction, possible complications or re-operations.

Statistical analysis was preformed using the unpaired Student's t-test with significance at $\mathrm{P}<0,05$ to compare patients with revision surgery to primary LRTI's (cohort history).

\section{RESULTS}

As shown in Table I., the results for revision surgery were compared to results of primary LRTI's. A statistical significant difference was seen for the DASH and Nelson score. The average VAS score was 2.9 (2.8 SD) on a scale of 10 .

Furthermore, we noted a good outcome for the opposition of the thumb based on the Kapandji score, with an average of 8.6 (1.4 SD) out of 10 . This was compared to the contralateral side, which scored 9,9 (0,4 SD), with a P value of 0.003 , making it statistically significant.

An overall loss of strength was mentioned by 12 patients, which correlated to the dynamometer scores : an average of $15.1 \mathrm{~kg}(9.7 \mathrm{SD})$ on the revised side and $20.5 \mathrm{~kg}$ (8.6 SD) con the contralateral side, with $\mathrm{p}=0.13$. Looking at the NHS questionnaire an average of 3.4 was evaluated for weakness of the thumb, going from 8 (none) to 0 (severe). Eight out of 14 patients experienced a sense of instability. However, increased laxity of the metacarpal base could only be withheld clinically in 2 patients and mildly in 2 others. The subjective evaluation of instability of the thumb correlated with a higher difference in strength between the operated side and the contralateral side; $6.1 \mathrm{~kg}$ (6.6 SD) versus 4.5 $\mathrm{kg}$ (6.9 SD) for patients with no sense of loss of stability.

Further clinical examination showed a preserved range of motion, without important shortcoming in any of the patients. There were sensory disturbances, mostly paresthesia, in 5 patients and a shoulder sign was seen in 3 .

The overall satisfaction concerning the revision was good for 8, fair for 3 and poor for another 3. Patients with a fair and poor outcome were disappointed because of strength loss, but pleased with the relief of pain they experienced preoperatively with the prosthesis.

Noteworthy is one female patient who was revised twice, with an additional Brunelli procedure between the first and second CMC joints after two unsuccessful corticosteroid infiltrations. All her scores were significantly worse compared to the average scores of the remaining 13 patients. She was the only patient who was displeased both in terms of power loss and pain.

\section{DISCUSSION}

The purpose of this study was to determine whether an interposition arthroplasty with ligament reconstruction is a successful salvage option for failed total joint prosthesis of the first CMC joint. We tried to evaluate the functional outcome of revision surgery and compared it to successful primary LRTI's. Results so far were rather promising, 
showing a VAS score similar to results obtained by Vandenberghe et al. (3) for primary LRTI's. We can also state that $79 \%$ of cases studied were considered satisfied with the reoperation. However, statistical significant differences were obtained for the Quick DASH score and NHS, showing deterioration of disability and impairment.

The Kapandji scores were acceptable after re-vision but significantly lower compared to the contralateral side, which in 9 out of 14 cases also contained a LRTI, prosthesis or surgically untreated arthritis. Whether this is an improvement of opposition is yet unclear, due to a lack of preoperative data. Vermeulen et al. (10) however showed significant improvement of opposition preand postoperatively of a primary Weilby arthroplasty, respectively 8.3 and 9.2 for the Kapandji score.

Hansen and Homilius (12) reported reduced grip strength up to less than $90 \%$ (75-90\%) compared to the other hand after revision with LRTI, giving similar results to the $26 \%$ strength loss we measured.

Although the results of the hydraulic dynamometer could only be compared to the contralateral side, it helped to quantify the sense of power loss experienced by $86 \%$ of patients. Ideally, grip force (and pinch grip) should be measured before and after placement of the prosthesis, for the initial procedure as well as the revision surgery, including long-term results. We have to take into consideration that untreated osteoarthritis of the thumb, as well as failed prostheses, are known to reduce grip strength $(13,14,15)$, and thus result in improved strength after treatment. Hence the results of a long-term study of Spekreijse et al. (16), comparing primary LRTI with or without bone tunnel after a mean follow-up of 5 years, obtained improved grip and pinch strength. Cooney et al. (17) measured grip strength before and after revision surgery and obtained improvement of power with $4 \mathrm{~kg}$ (average of $16 \mathrm{~kg}$ before and $20 \mathrm{~kg}$ after). The latter study however excluded all primary prosthesis.

Knak et al. (18) compared the outcome of revision using trapeziectomy with revision into a cemented trapezium cup, proving a higher complication rate in the second group. They concluded trapeziectomy should be the standard revision technique giving the unacceptable failure rate of a cemented cup. Lenoir et al. also confirms LRTI to be an attractive salvage procedure.

Alternative revision surgeries such as scaphometacarpal arthroplasties described by Chiche et al. (19) showed rather promising results in 8 out 9 patients. More research is needed to determine if this could be a better salvage option, taken into consideration risks of implant failure. So far, no evidence was found that scaphometacarpal prosthesis provide a better clinical outcome.

Arthrodesis with an autologous corticocancellous iliac crest bone graft after multiple revisions, a case report by Goorens et al. (20), was proven to be a feasible alternative for treatment of failed trapeziectomy based on pain relief. Primary arthrodesis and LRTI's have similar functional outcome $(7,21)$, but no literature could be found on revision surgeries comparing both.

Results of Munns et al. (22), comparing primary surgery to revision, correlates with ours. They also noted that patients with associated metacarpal phalangeal joint pathology addressed are more likely to have better results.

Ardouin et al. (23) described a technique for significant bone loss of the first metacarpal, where they molded an iliac crest bone graft and preformed a capsular reconstruction using a section of the FCR, restoring the length of the first metacarpal with additional stability. They obtained good results, however grip strength was not measured.

Remaining options consist of silicone implants and spacers. No literature was found for the use of silicone implants as a salvage option. Cootjans et al. (24) showed that spacers have clinically good results, but no major benefits where withheld compared to LRTI's.

Prospective long-term studies with measured clinical data are needed for better evaluation of revision surgery for failed total joint prosthesis.

\section{CONCLUSION}

Revision surgery with LRTI can be considered a good salvage option for failed total joint prosthesis with good improvement in pain relief and mobility in $79 \%$ cases. However, slightly more impairment and disability should be taken into account as 
compared to primary cases. Subjective and objective evaluations indicate strength loss in $86 \%$, yet only $25 \%$ of them were unsatisfied because of it.

\section{REFERENCES}

1. Berger A, Meals R. Surgical treatment of carpometacarpal arthritis. Curr Orthop Pract. 2014 Oct ; 25 : 420-425.

2. Warwick D, Beredjiklian P, De Smet L, Smit A, Vermeulen G. IFSSH Scientific Committee on Degenerative Arthritis - CMC Joint, 2014 Mar ; 1-12.

3. Vandenberghe L, Degreef I, Didden K, Fiews S, De Smet L. Long term outcome of trapeziectomy with ligament reconstruction/tendon interposition versus thumb basal joint prosthesis. J. Hand Surg. Eur. 2014 Apr ; 39 : 118.

4. Croog A, Rettig M. Newest Advances in the operative treatment of basal joint arthritis. Bull. NYU Hosp. Jt. Dis. $2007 ; 65: 78-86$.

5. Heyworth BE, Jobin CM, Monica JT, Crow SA, Lee JH, Rosenwasser MP. Long-Term Follow-Up of Basal Joint resection Arthroplasty of the Thumb With Transfer of the Abductor Pollicis Brevis Origin to the Flexor Carpi Radialis Tendon. J. Hand Surg. 2009 Jul-Aug ; 34A : 10211028.

6. Vermeulen GM, Slijper H, Feitz R. et al. Surgical Management of Primary Thumb Carpometacarpal Osteoarthritis : A Systematic Review. J. Hand Surg. 2011 Jan ; 36A : 157-169.

7. Ghavami A, Oishi SN. Thumb Trapeziometacarpal Arthritis : Treatment with Ligament Reconstruction Tendon Interposition Arthroplasty. Plast. Reconstr. Surg. 2006 May; 117 : 116e-128e.

8. Matullo KS, Ilyas A, Thoder JJ. CMC Arthroplasty of the thumb : A review. Hand (NY) 2007 Aug ; 2 : 232-239.

9. Weilby A. Tendon Interposition Arthroplasty of the first carpo-metacarpal joint. J. Hand Surg. 1988 Nov ; 3B : 421425

10. Kapandji A. [Clinical test of apposition and counterapposition of the thumb]. Ann. Chir. Main 1986 ; 5 : 67-73.

11. Citron, N., Hulme, C., \& Wardle, N. A self-administered questionnaire for basal osteoarthritis of the thumb. J. Hand Surg. Eur. 2007 ; 32(5) : 524-528.

12. Hansen TB, Homilius M. Failed total carpometacarpal joint prosthesis of the thumb: Resuls after resection arthroplasty. J. Plast. Surg. Hand Surg. 2010 Jun ; 44 : 171174.
13. Vermeulen GM, Brink SM, Sluiter J, Elias SG, Hovius SE, Moojen TM. Ligament Reconstruction Arthroplasty for Primary Thumb Carpometacarpal Osteoarthritis (Weilby Technique) : Prospective Cohort Study. J. Hand Surg. 2009 Sept ; 34A : 1393-1401.

14. Tomaino MM, Pellegrini VD, Burton RI. Arthroplasty of the basal joint of the thumb : long-term follow-up after ligament reconstruction with tendon interposition. J. Bone Joint Surg. Am. 1995 Mar ; 77 : 346-355.

15. Trumble T, Rafijah G, Heaton D. Thumb Carpometacarpal Arthroplasty with Ligament Reconstruction and Interposition Costochondral Arthroplasty. J. Wrist Surg. 2013 Aug ; $2: 220-227$.

16. Spekreijse KR, Vermeulen GM, Kedilioglu MA et al. The Effect of Bone Tunnel During Ligament Reconstruction for Trapezoimetacarpal Osteoarthritis : A 5-year Follow-up. J. Hand Surg. Am. 2015 Nov ; 40 : 2214-2222.

17. Cooney WP, Leddy TP, Larson DR. Revision of Thumb Trapeziometacarpal Arthroplasty. J. Hand Surg. 2006 Feb ; 31A : 219.e1-10.

18. Knak J, Hansen TR. Trapeziectomy or revision into cemented polyethylene cup in failed trapeziometacarpal total joint arthroplasty. Plast. Surg. Hand Surg. 2016 Oct ; 50(5) : 286-290.

19. Chiche L, Lamarre H, Barbary S, Teissier J. Scaphometacarpal arthroplasty : a report of ten cases of trapeziometacarpal prosthesis and trapeziectomy revison. J. Hand Surg. Eur. 2020 Jan ; 0(0) : 1-5.

20. Goorens CK, Van Schaik DE, Goubau JF. Surgical treatment after a failed trapeziectomy : A case report. Chir. Main, 2015 Sep ; 34 : 205-209.

21. Hart R, Janecek M, Siska V, Kucera B, Stipcak V. Interposition suspension arthroplasty according to Epping versus arthrodesis for trapeziometacarpal osteoarthritis. Eur. Surg. Acta Chirurgica Austriaca 2006 Dec ; 38 : 433438.

22. Munns JJ, Matthias RC, Zarezadeh A. et al. Outcomes of revisions for failed trapeziometacarpal joint arthritis surgery. J. Hand Surg. Am. 2019 Sep ; 44(9) : 798.e1-798. e9.

23. Ardouin L, Mattelaer B, Villani F, Vaienti L, Merle M. Reconstruction for failed trapeziometacarpal implant. Tech. Hand Surg. 2011 Dec ; 15 : 225-229.

24. Cootjans K, Dreessen P, Vandenberghe D, Verhoeven N. Salvage revision arthroplasty after failed TMC joint prosthesis. Acta Orthop. Belg. 2019 Sep ; 85 : 325-329. 


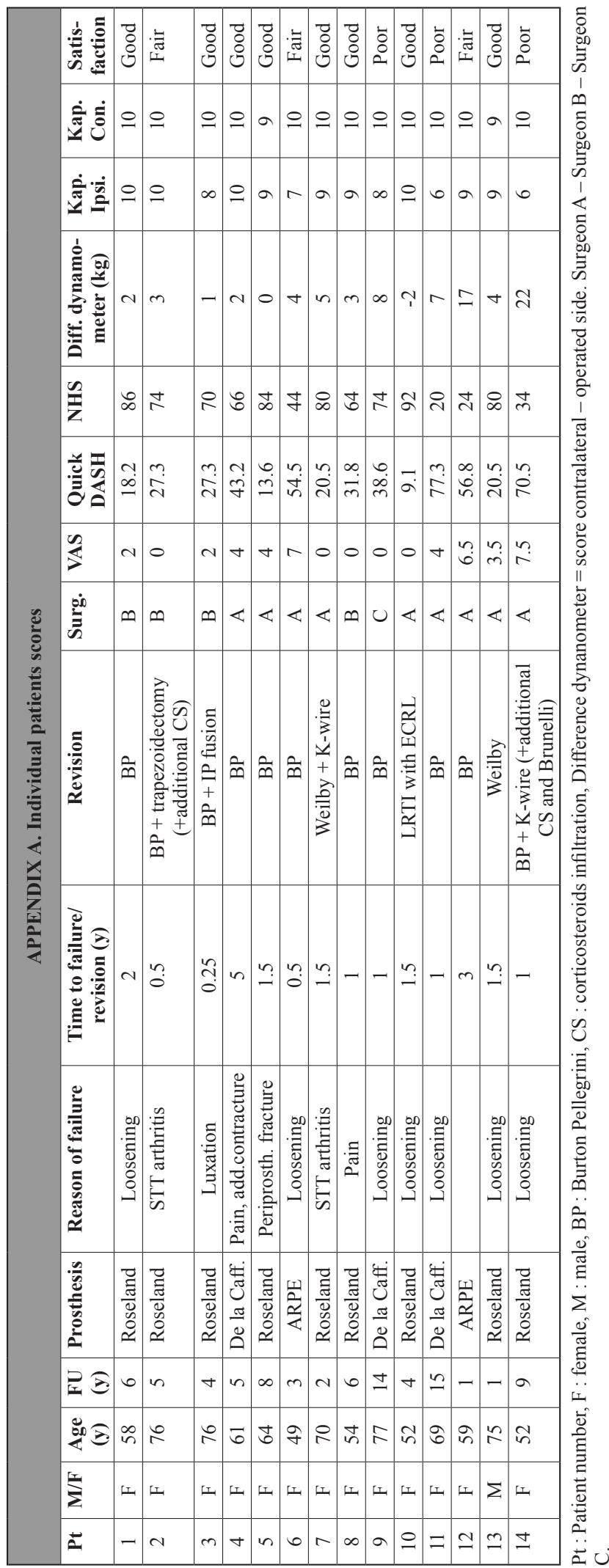

